\title{
Calculations on the optical properties of the elliptical and cubical nanoparticles with electrostatic approach Chunzhen Fan ${ }^{1}$, , , Shuangmei-Zhu ${ }^{2, b}$ \\ ${ }^{1}$ School of Physical Science and Engineering, Zhengzhou University, Zhengzhou 450052, China \\ ${ }^{2}$ College of Science, Henan Institute of Engineering, Zhengzhou, 451191, China

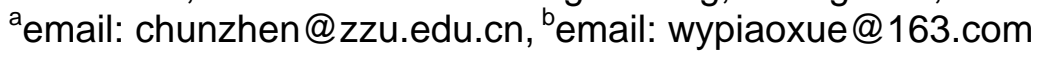

Keywords: elliptical nanoparticles; cubic nanoparticles; electrostatic approach

\begin{abstract}
Metallic nanoparticles display unique properties that are quite different from those of individual atoms. In reality, the optical properties of the nanoparticles strongly depend on its shape. We present the electrostatic approach to evaluate scattering and absorption of different shaped nanoparticles under an oscillating electric dipole moment. For numerical simulations, we present the depolarization factor of the elliptical and cubical nanoparticles with a Drude dielectric profile. Numerical results illustrate that the geometry factor, the host dielectric constant and the damping coefficient can affect the scattering and absorption spectrum significantly. To meet the requirements of optical devices, such a tunable structure can be used to design optical sensors, modulators and detectors.
\end{abstract}

\section{Introduction}

Metallic nanoparticles with their fascinating properties have attracted much attention in recent years [1]. Due to their small size effect and tunable surface plasmon resonance, these particles yield a wide range of applications in chemical and biological sensors, plasmonic system and so on [2]. The optical properties of metal nanoparticles can be engineered by their size, shape and dielectric environment [3]. Control over the plasmon frequencies has been gained by playing with different shapes, as recently shown for nanowires, nanorings, nanorods, nanorice, nanostar and nanocages even the nanoparticles array [4]. To obtain these nanoparticles, we can resort to the seed-mediated method [5], the polyol method at different reaction temperature [6], the biological method [7] and the chemical reduction method [8]. The formation of metallic nanoparticles involves solvent, metal ions and copolymers. The copolymers have both reducing components to form stabilizing components. The method can be used to form metal nanoparticles having a desired shape and size. Experimental mechanism of shape evolution of metallic nanoparticles has been systematically studied in Ref. $[9,10]$. Theoretically, the Mie theory has been widely used in the calculation of the absorption and scattering of the nanoparticles which is a tedious work with numerically iteration. [11] Here we use the electrostatic approach to calculate the optical properties of the nanoparticles in a much easier way.

One important factor governing the various shapes of nanoparticles embodying the fascinating properties is the geometry factor. We have listed out the geometry factor of the spherical, elliptical and cubical nanoparticles respectively. Then the depolarization has been obtained with the static electromagnetic theory with the incident plane wave along the $z$ direction. We numerically investigate the shape effect on the scattering and absorption cross section of the spherical, elliptical and cubical nanoparticles, whose dielectric constant observe the Drude form. By tuning the host dielectric constant, the damping efficiency and the geometry factor, the optical properties are fully explored with the electrostatic approach.

\section{Formulism}

Let us start by considering a spherical metallic particle with radius $a$ suspended in the host medium with dielectric constant $\varepsilon_{m}$. The dielectric response of the sphere is further described by the dielectric function $\varepsilon(\omega)$, which we take as a simple complex number. The external electric field is along the $z$ axis of the form $E=E_{0}(z)$. It is well known that the relative permittivity of metals can be described by means of the Drude model as following [12] $\varepsilon(\omega)=\varepsilon_{\infty}-\frac{\omega_{p}^{2}}{\omega(\omega+i \gamma)}$. The first 
material parameter $\varepsilon_{\infty}$ for all dielectric dispersions models is the epsilon infinity value, representing the high frequency limit of the permittivity. $\omega_{p}$ is the plasma frequency and $\gamma$ is the damping coefficient. $\omega=2 \pi c / \lambda$ is the discrete values of the frequency. In addition, we set $\gamma=0.02 \omega_{p}$ typical value for the noble bulk metals, $\varepsilon_{2}$ dielectric constant of the glass.

In the electrostatic approach, we are interested in a solution of the Laplace's equation for the potential inside and outside the spherical particles. The derivation is a standard textbook problem in the spherical coordinates. Due to the azimuthal symmetry of the problem, the potential inside and outside the sphere is given by respectively [13]

$$
\begin{aligned}
& \varphi_{\text {in }}(\mathrm{r}, \theta)=\sum_{l=0}^{\infty} A_{l} r^{l} P_{l}(\cos \theta) \\
& \varphi_{\text {out }}(\mathrm{r}, \theta)=\sum_{l=0}^{\infty}\left[B_{l} r^{l}+C_{l} r^{-(l+1)}\right] P_{l}(\cos \theta)
\end{aligned}
$$

The coefficients $\mathrm{A}_{l}, \mathrm{~B}_{l}$ and $\mathrm{C}_{l}$ can be determined from the boundary conditions at $r$ is infinite and at the sphere surface $(\mathrm{r}=\mathrm{a}) . \quad P_{l}(\cos \theta)$ is the Legendre functions. With boundary conditions, the continuity of the tangential components of the electric field $E=-\nabla \varphi$ demands that $\left.\frac{\partial \varphi_{\text {in }}}{\partial \theta}\right|_{r=a}=\left.\frac{\partial \varphi_{\text {out }}}{\partial \theta}\right|_{r=a}$; The continuity of the normal components of the displacement field requires that $\left.\varepsilon \frac{\partial \varphi_{\text {in }}}{\partial \theta}\right|_{r=a}=\left.\varepsilon_{m} \frac{\partial \varphi_{\text {out }}}{\partial \theta}\right|_{r=a}$.Solving the above equation, we obtain

$$
\begin{aligned}
& \varphi_{\text {in }}=-\frac{3 \varepsilon_{m}}{\varepsilon+2 \varepsilon_{m}} E_{0} r \cos \theta \\
& \varphi_{\text {out }}=-E_{0} r \cos \theta+\frac{\varepsilon-\varepsilon_{m}}{\varepsilon+2 \varepsilon_{m}} E_{0} a^{3} \frac{\cos \theta}{r^{2}}
\end{aligned}
$$

Under plane-wave illumination, the fields induce an oscillating dipole moment. The radiation of this dipole leads to scattering of the plane wave by the sphere, which can be represented the radiation by a point dipole. We can rewrite $\varphi_{\text {out }}$ by introducing the dipole moment $\mathbf{p}$ as

$$
\begin{aligned}
& \varphi_{\text {out }}=-E_{0} r \cos \theta+\frac{\mathbf{p} \cdot r}{4 \pi \varepsilon_{0} \varepsilon_{m} r^{3}} \\
& \mathbf{p}=4 \pi \varepsilon_{0} \varepsilon_{m} a^{3} \frac{\varepsilon-\varepsilon_{m}}{\varepsilon+2 \varepsilon_{m}} E_{0}
\end{aligned}
$$

Introduce the polarizability $\alpha$, which was defined via $\mathbf{p}=\varepsilon_{0} \varepsilon_{m} \alpha \mathrm{E}_{0}$. Thus, $\alpha=4 \pi \mathrm{a}^{3} \frac{\varepsilon-\varepsilon_{\mathrm{m}}}{\varepsilon+2 \varepsilon_{\mathrm{m}}}$.

The above equation describes the polarizability of a small sphere of sub-wavelength diameter in the electrostatic approximation. The depolarization factor is a descriptor of the extent to which the inclusion polarization is diminished according to the particle's shape and orientation with respect to the applied electrical field. For the spherical nanoparticles, its symmetry is along three directions, thus the geometry factor is $1 / 3$.

However, it has to be pointed out that geometry effect on the nonspherical nanoparticles, like the elliptical and cubical particles. Generally, ellipsoid having axial dimensions a, b, and c can be described in Cartesian coordinates as $\frac{x^{2}}{u+a^{2}}+\frac{y^{2}}{u+b^{2}}+\frac{z^{2}}{u+c^{2}}=1$ where $x, y$, and $z$ are the position coordinates of any point on the surface of the ellipsoid. Here we take $G^{i}$ to be the depolarization factor in the $i$ th axial direction which is given as [4] $G^{i}=\int_{0}^{\infty} \frac{(a b c) d u}{2\left(u+i^{2}\right) \sqrt{\left(u+a^{2}\right)\left(u+b^{2}\right)\left(u+c^{2}\right)}}$.

The geometry factor along three directions satisfy the relation $\sum_{i=1}^{3} G^{i}=1$. For the prolate and oblate case $(\mathrm{b}=\mathrm{c})$, the depolarization factor can be simplified as $G^{a}=\frac{1}{1+1.6 \times\left(\frac{a}{b}\right)+0.4 \times\left(\frac{a}{b}\right)^{2}}$, $G^{b}=G^{c}=\frac{1-G^{a}}{2}$. For the spherical case $(a=b=c)$, the depolarization factors of a sphere $G^{a}=G^{b}=G^{c}=1 / 3$. The depolarization factors vary depending on the volume ratio of each set of confocal ellipsoids. The corresponding cross sections for scattering and absorption Qsca and Qabs can be expressed as followings

$$
\begin{aligned}
& \sigma_{\text {sca }}=\frac{k^{4} V_{p}^{2}}{6 \pi} \sum_{j=1}^{3} \frac{1}{3}\left|\frac{\varepsilon-\varepsilon_{m}}{\varepsilon_{m}+G_{j}\left(\varepsilon-\varepsilon_{m}\right)}\right|^{2} \\
& \sigma_{\mathrm{abs}}=k V_{\mathrm{p}} \operatorname{Im} \sum_{j=1}^{3} \frac{1}{3}\left(\frac{\varepsilon-\varepsilon_{m}}{\varepsilon_{m}+G_{j}\left(\varepsilon-\varepsilon_{m}\right)}\right)
\end{aligned}
$$

With the calculation of the poynting-vector, the corresponding cross sections for scattering and 
absorption $Q_{\text {sca }}$ and $Q_{a b s}$ can be expressed as followings, where $\sigma_{\text {sca }}=\frac{Q_{\text {sca }}}{\pi a^{2}}, \sigma_{\mathrm{abs}}=\frac{Q_{\mathrm{abs}}}{\pi a^{2}} . \sigma_{\mathrm{sca}}$ and $\sigma_{\text {abs }}$ are the scattering and absorption efficiency respectively. The extinction cross section of a silver sphere in the quasi-static approximation satisfies the formula $Q_{\text {ext }}=Q_{\mathrm{abs}}+Q_{\mathrm{sca}}$.

According to Fuchs, the dipolar polarizability of a cubic particle is composed of $N$ dipolar eigenmodes of the induced charge distribution in the cubic particle around the edges and corners which are always excited simultaneously. For the cubic particles, normal resonant modes correspond to the six major absorption peaks of a cube. These six eigenmodes have together an oscillator strength of $G(m)=0.96$, only $4 \%$ of all oscillator strength belongs to eigenmodes not shown here. As we now have six different geometry factors $G(m)$. The values of the depolarization factors $n_{\mathrm{m}}$ and the constants $G(m)$ or these six major resonances are given in Ref. [14, 15]. $\alpha=\frac{1}{4 \pi} \sum_{m} \frac{G(m)}{\left(\frac{\varepsilon}{\varepsilon_{2}}-1\right)^{-1}+n_{m}}$. Thus, the scattering efficiency and absorption efficiency can be written as,

$$
\sigma_{\text {sca }}=\frac{k^{4} V_{p}}{6 \pi}|\alpha|^{2}
$$

$\sigma_{a b s}=4 \pi k V_{p} \operatorname{Im}[\alpha]$

Where $V_{p}$ is the volume of the cubic nanoparticles. It equals $a^{3}$, $a$ is the side length of the nanoparticles.

\section{Numerical results}

Parameters governing the features of optical properties of the various shaped materials have been calculated with the electrostatic approach. The numerical results are shown in figure 1-5.In figure 1, we have calculated the scattering efficiency of the elliptical nanoparticles with different damping factor $\gamma$ as a function of the incident frequency. Here the varied damping factor represents different metals. The host dielectric environment is the glass and $\varepsilon_{2}=1.5$.
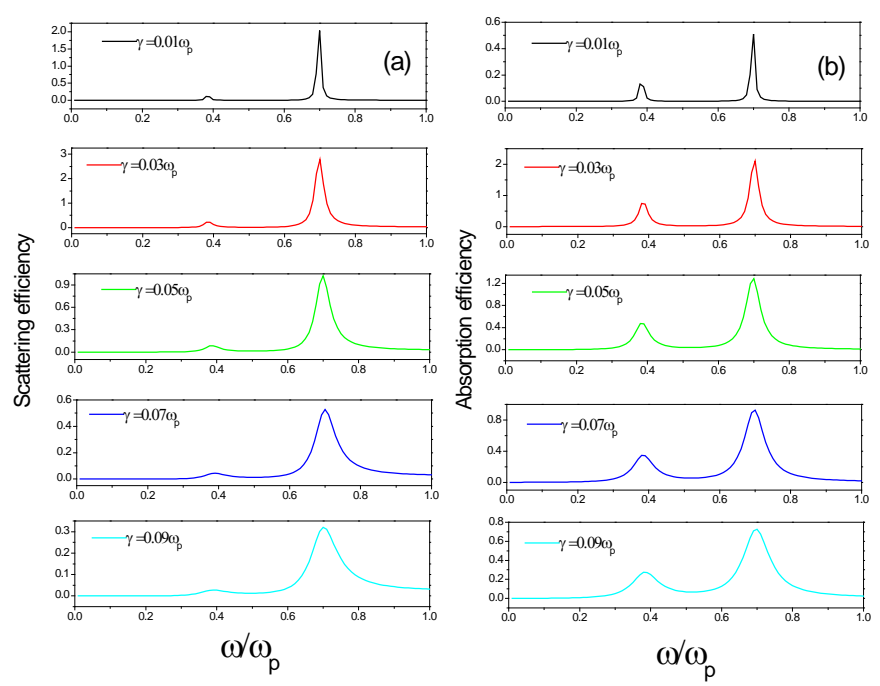

Figure 1. The scattering efficiency (a) and absorption efficiency (b) of the elliptical nanoparticles with different damping factor $\gamma$. Parameters: $b=c, a / b=0.4, \varepsilon_{2}=1.5$.

For the ellipsoid nanoparticles, we take $b=c$, and $x=a / b=0.4$ in our numerical calculations for simplicity, the scattering and absorption efficiency of oblate elliptical nanoparticles were investigated in figure 1 and figure 2. Due to the symmetry along the $b$ and $c$ axis, there are two resonant peaks and they got broadened with the increased damping factor, which was shown in figure 1. When the host dielectric constant was increased from 1.0 to 5.0 in figure 2, the resonant peaks shall move to the low frequency region, namely, there is a redshift. 


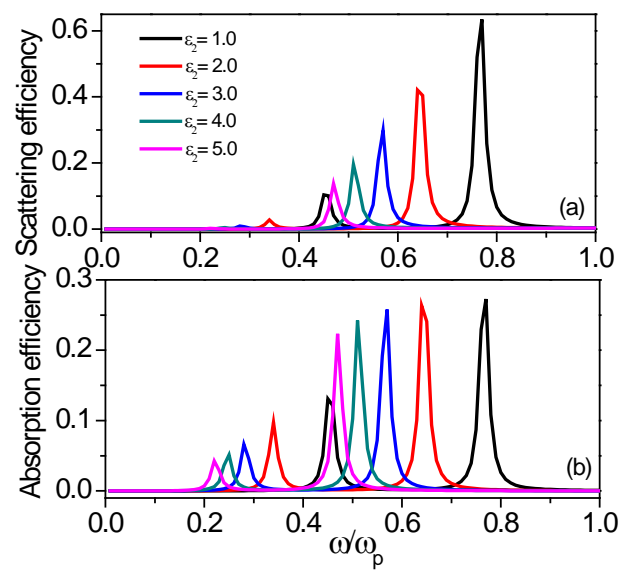

Figure 2. The scattering (a) and absorption (b) efficiency of the elliptical nanoparticles with different $\varepsilon_{2}$. Parameters: $b=c, a / b=0.4, \gamma=0.02 \omega_{p}$

For the elliptical nanoparticles, $\mathrm{x}$ represents the axis ratio and it equals $\mathrm{a} / \mathrm{b}$. When $\mathrm{x}$ is smaller than 1.0, it means the prolate nanoparticles. $x$ is larger than 1 indicates its oblate nanoparticles. When $\mathrm{x}=1.0$, it represents the spherical nanoparticles, with $\mathrm{a}=\mathrm{b}=\mathrm{c}$. Here the geometry effect on the

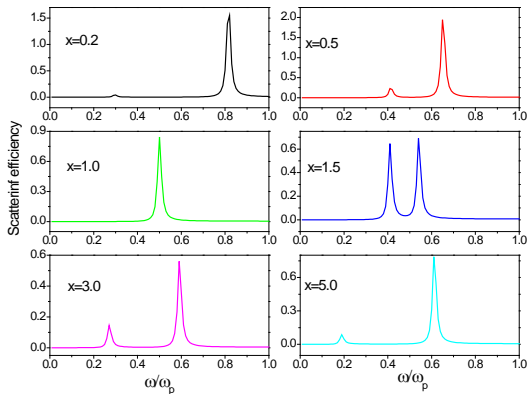

(a)

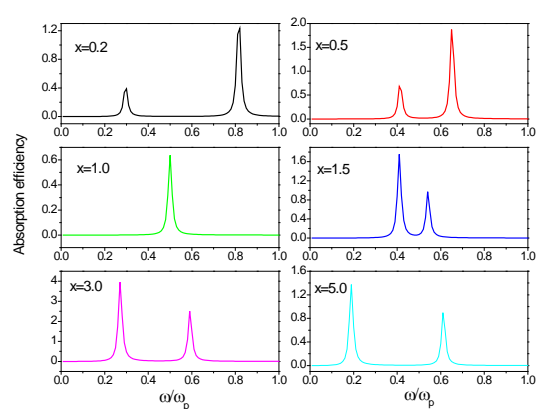

(b)

Figure 3. The scattering (a) and absorption (b) efficiency of the elliptical nanoparticles with different axis ratios $x=a / b$. Parameters: $\varepsilon_{2}=1.5, \gamma=0.02 \omega_{p}$

scattering and absorption spectra was shown in figure 3, where two resonant peaks show a trade off with the increased value of $\mathrm{x}$. The lower frequency peak got enhanced with the larger $\mathrm{x}$, while the high frequency resonant peak decreased with the larger $\mathrm{x}$.
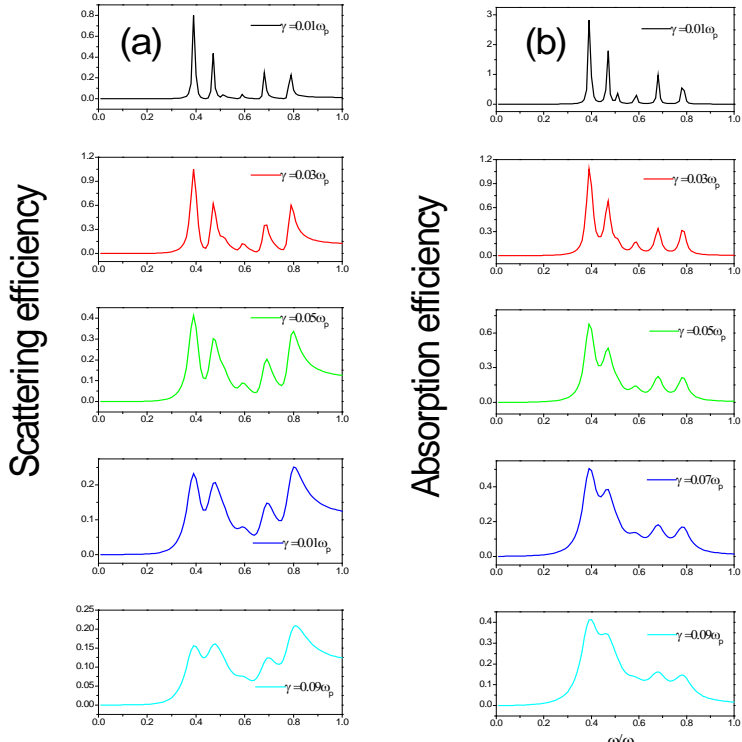

Figure 4. The scattering (a) and absorption (b) efficiency of the cubical nanoparticles with different damping factor $\gamma$. Parameters: $\varepsilon_{2}=1.5, a=30 \mathrm{~nm}$. 
For the cubical nanoparticles, the scattering and absorption efficiency was shown in figure 4 and figure 5. The length of each side is taken as $a=30 \mathrm{~nm}$. Six resonant peaks can be clearly observed with different damping factor and the host dielectric constant. And these resonant peaks can be tuned accordingly.

\section{Conclusions}

The optical properties of the light interacting with different shaped metallic nanoparticles were assessed with static electromagnetic method. By evaluating the geometry factor of the spherical, elliptical and cubical nanoparticles, the absorption and scattering spectra were obtained respectively. And the tunable resonant peaks have also been studied by varying the damping factor and the dielectric constant of the host medium. Numerical results illustrate that these resonant peaks can be tuned as desired, which give us a guideline in the future design of the nano-electronic devices.
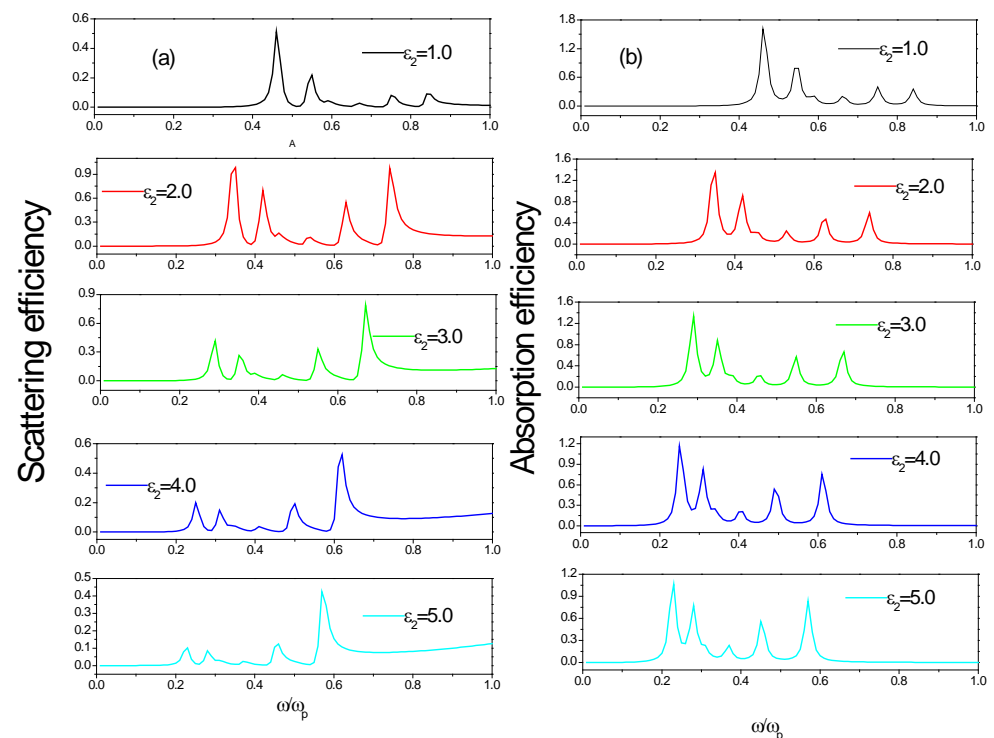

Figure 5. The scattering (a) and absorption (b) efficiency of the cubical nanoparticles with different dielectric constant $\varepsilon_{2}$. Parameters: $\gamma=0.02 \omega_{p}, a=30 \mathrm{~nm}$.

\section{Acknowledgement}

This work is supported by the Key science and technology research project of Henan Province (142102210489), by the Foundation of Henan Educational Committee (14A140004).

†Corresponding author. E-mail: chunzhen@zzu.edu.cn

\section{References}

[1] Bohren C F and Huffman D R 1998 Absorption and Scattering of Light by Small Particles (Wiley-Interscience Publication, John Wiley \& Sons)

[2] Mie and Beyond 2011 Optical Properties of Nanoparticle Systems (Michael Quinten Wiley)

[3] Kelly K L, Coronado E, Zhao L L and Schatz G C 2003 J Phys. Chem. B 107668

[4] Jones S B and Friedman S P 2000 Water Resour. Res. 362821

[5] Personick M L, Langille M R, Zhang J and Mirkin C A 2011 Nano Lett. 113394

[6] Mustafa S Y, Xia Y N et al. 2009 Nature Mater. 8935

[7] Punjabi K, Choudhary P, Samant L, Mukherjee S, Vaidya S and Chowdhary A 2015 Int. J. Pharm. Sci. Rev. Res. 40219

[8] Kulkarni N and Muddapur U 2014 J. Nanotech. 510246 
[9] Paschalis A and Toshio S 2010 United States Patent 7718094

[10]Fan C Z, Wang J Q, He J N, Ding P and Liang E J (2013) Chin. Phys. B 22074211

[11]Li H Y, Wu Z S and Li Z J 2010 Chin, Phys. B 10277

[12] Vial A, Grimault A S, Macías D, Barchiesi D and Chapelle M L 2005 Phys. Rev. 71085416

[13]Huang J P and Yu K W 2006 Phys. Rep. 4313

[14]Fuchs R 1975 Phys. Rev. B 111732

[15]Ruppin R Z 1996 Phys. D 3669 